\title{
HAUSDORFF METHODS OF SUMMATION AND CONTINUED FRACTIONS
}

\author{
BY \\ H. L. GARABEDIAN AND H. S. WALL
}

1. Introduction. We shall be occupied in this paper with a special study of the transformation

$$
t_{m}=\sum_{n=0}^{m} a_{m n} s_{n}, \quad \quad m, n=0,1,2, \cdots,
$$

where $\left\{s_{n}\right\}$ is an infinite sequence of numbers. If, in particular, we are concerned with the infinite series $\sum_{\nu=0}^{\infty} u_{\nu}$, then $s_{n}=\sum_{\nu=0}^{n} u_{\nu}$. Let $\mathfrak{A}=\left(a_{m n}\right)$ represent the triangular matrix of the transformation (1.1). Then, the sequence $\left\{t_{m}\right\}$ is called the transform of $\left\{s_{n}\right\}$ by the matrix $\mathfrak{A}$ and is represented in symbolic form by $t_{m}=\mathfrak{A}\left\{s_{n}\right\}$. If the matrix $\mathfrak{A}$ is regular, in the sense of Silverman [1] and Toeplitz [2], then the matrix $\mathfrak{A}$ defines a regular method of summation (summability).

In this paper our attention is focused on a class of regular and permutable matrices $\{\mathfrak{A}\}$, known as Hausdorff matrices ([3] or [4]), which we shall presently define. Let $\left\{c_{m}\right\}$ be an infinite sequence of numbers defined by the Stieltjes integrals

$$
c_{m}=\int_{0}^{1} u^{m} d \phi(u), \quad m=0,1,2, \cdots .
$$

Then, we form the matrix $\mathfrak{D}\left(\delta_{m n} c_{m}\right) \mathfrak{D}$, where $\delta_{m n}=0$ for $m \neq n, \delta_{m m}=1$, and $\mathfrak{D}=\left((-1)^{n} C_{m, n}\right)$. Incidentally, the matrix $\mathfrak{D}$ has the property $\mathfrak{D}^{2}=\mathfrak{\Im}$, where $\Im$ is the identity matrix. The following conditions on the mass function $\phi(u)$ are necessary and sufficient in order that the matrix $\mathfrak{D}\left(\delta_{m n} c_{m}\right) \mathfrak{D}$ be regular:

(1.3) (i) $\phi(u)$ is of bounded variation on the closed interval $(0,1)$;

(ii) $\phi(u)$ is continuous at $u=0$ and $\phi(u)=0$;

(iii) $\phi(1)=1$;

(iv) $\phi(u)=\frac{1}{2}[\phi(u-0)+\phi(u+0)], 0<u<1$.

If $\phi(u)$ satisfies the conditions (1.3), then the matrix $\mathfrak{D}\left(\delta_{m n} c_{m}\right) \mathfrak{D}$ is a Hausdorff matrix and the sequence (1.2) is known as a regular sequence or a regular moment sequence. If $\mathfrak{A}=\mathfrak{D}\left(\delta_{m n} c_{m}\right) \mathfrak{D}$ Hausdorff has proved that we can write

$$
a_{m n}=C_{m, n} \Delta^{m-n} C_{n} \text {. }
$$

Presented to the Society, April 13, 1940; received by the editors February 2, 1940, and, in revised form, February 24, 1940. 
We shall be concerned for the most part with real totally monotone sequences $\left\{c_{n}\right\}$ which are characterized in any one of the following three ways:

(1.5) (i) $\Delta^{m} c_{n} \geqq 0,(m, n=0,1,2, \cdots)$;

(ii) there exists (essentially uniquely $\left.\left({ }^{1}\right)\right)$ a real monotone non-decreasing $\phi(u)$ such that $c_{n}=\int_{0}^{1} u^{n} d \phi(u),(n=0,1,2, \cdots)$;

(iii) there is a correspondence $\left(^{2}\right)$ of the form

$$
c_{0}-c_{1} x+c_{2} x^{2}-\cdots \sim \frac{c_{0}}{1}+\frac{g_{1} x}{1}+\frac{\left(1-g_{1}\right) g_{2} x}{1}+\frac{\left(1-g_{2}\right) g_{3} x}{1}+\cdots,
$$

where $c_{0} \geqq 0,0 \leqq g_{n} \leqq 1,(n=1,2,3, \cdots)$, and where it is understood that the continued fraction shall terminate with the first partial quotient which vanishes identically.

These characterizations are due respectively to I. Schur, F. Hausdorff [5], and H. S. Wall [6].

In $\$ 2$ of this paper we give necessary and sufficient conditions for the regularity of a totally monotone sequence in terms of the corresponding continued fraction. In $\$ 3$ we investigate certain properties of the difference matrix $\Delta=\left(\Delta^{m} c_{n}\right)$. Any row, column, or diagonal sequence of the difference matrix is found to be totally monotone. We obtain necessary and sufficient conditions, in terms of the continued fraction corresponding to the base sequence, that is, the sequence defined by the first row of $\Delta$, in order that the row, column, and diagonal sequences be regular. This is accomplished with the aid of the following curious result: if $(1.5$, iii) obtains, then the power series $c_{0}-\Delta c_{0} x+\Delta^{2} c_{0} x^{2}-\cdots$, with coefficients in the first column of the difference matrix $\Delta$, corresponds to the continued fraction

$$
\frac{c_{0}}{1}+\frac{\left(1-g_{1}\right) x}{1}+\frac{g_{1} g_{2} x}{1}+\frac{\left(1-g_{2}\right)\left(1-g_{3}\right) x}{1}+\frac{g_{3} g_{4} x}{1}+\cdots,
$$

obtained from the continued fraction of $\left(1.5\right.$, iii) by replacing $g_{2 n-1}$ by $1-g_{2 n-1}$, $(n=1,2,3, \cdots)$. Section 4 is devoted to an example which illustrates some of the results of the two preceding sections.

The remainder of this paper has to do with special Hausdorff methods of summation. In $\$ 5$ the continued fraction of Gauss is employed to obtain a regular sequence which results in the definition of hypergeometric summability. Numerous inclusion and equivalence relations between the hypergeometric methods are derived. In $\$ 6$ we replace the base sequence of $\Delta$ by known se-

(1) This means that $\phi(u)$ exists uniquely except for an additive constant at all points of continuity.

(2) We use the symbol $\sim$ between a power series and a continued fraction to indicate that the power series expansion of the $n$th approximant of the continued fraction agrees term by term with the given power series for more and more terms as $n$ is increased, or becomes identical with it from and after some $n$. 
quences and discuss the new methods of summability thus generated and some of their properties. Finally, in $\$ 7$, we discuss the effectiveness of methods of summation associated with regular totally monotone sequences relative to the analytic continuation of power series outside the circle of convergence.

2. The Stieltjes continued fraction as a tool in the theory of summability. In his celebrated memoir Recherches sur les fractions continues, Stieltjes [7] showed that a real sequence $\left\{c_{n}\right\}$ is a moment sequence for an infinite distribution of mass along the positive half of the real axis if and only if the power series $c_{0}-c_{1} x+c_{2} x^{2}-\cdots$ has a corresponding continued fraction of the form $b_{1} / 1+b_{2} x / 1+b_{3} x / 1+\cdots$, where the $b_{n}$ 's are real and positive. The continued fraction is uniquely determined by the moment sequence, and conversely there is uniquely determined a moment sequence by means of a continued fraction of the specified form. Moreover, the question as to the uniqueness of the distribution of mass for a given moment sequence can always be decided when the continued fraction is known.

When the continued fraction converges, Stieltjes showed that the function represented has the form

$$
\int_{0}^{\infty} \frac{d \phi(u)}{1+x u}
$$

where $\phi(u)$ is a bounded monotone non-decreasing function, and represents the distribution of mass in accordance with the relations $c_{n}=\int_{0}^{\infty} u^{n} d \phi(u)$, $(n=0,1,2, \cdots)$, the sequence $\left\{c_{n}\right\}$ defining the given moments. In this case the moment problem is determinate, that is, there is but one possible distribution of mass. On the other hand, if the continued fraction diverges, the sequences of even and odd approximants converge to separate integrals of the above form, which determine two distinct solutions of the moment problem. In this, the indeterminate case, there are infinitely many distributions of mass for the given moments.

Until recently it was not known how to specialize the numbers $b_{n}$ in the continued fraction in the case of moments for a distribution of mass over the finite interval $(0,1)$. The answer to this question is contained in the statement $(1.5$, iii), where, in the case of the terminating continued fraction, the moments are for a finite distribution of mass.

The moment problem for the interval $(0,1)$ was solved without the use of continued fractions by Hausdorff [3]. He found, among other things, that these sequences are regular $\left({ }^{3}\right)$ if and only if the mass function $\phi(u)$ of $(1.5$, ii) is continuous at $u=0$, and $\int_{0}^{1} d \phi(u)=c_{0}=1$. One of the ways in which the con-

$\left.{ }^{3}\right)$ It is sometimes convenient to state the regularity conditions (1.3) in this form. The requirements $\phi(0)=0, \phi(1)=1$ can be replaced by the single condition $\int_{0}^{1} d \phi(u)=1$. In the discussion which follows we shall always assume without further mention that this requirement is met. The regularity condition $(1.3, \mathrm{iv})$ is in a sense superfluous since it serves merely to determine $\phi(u)$ uniquely at every point of the interval $(0,1)$. 
tinued fraction may serve as a tool in this theory is in establishing the continuity or the discontinuity of $\phi(u)$ at $u=0$.

We shall begin by disposing of the case where the continued fraction (1.5, iii) terminates, the case corresponding to a finite distribution of mass. Here the function $\phi(u)$ is a step function with but a finite number of discontinuities, one at each point where a quantity of mass is concentrated. There is no discontinuity at $u=0$ if and only if for some index $n$ the first $2 n$ partial quotients of the continued fraction are not identically zero while the next partial quotient vanishes identically. This [6] is a consequence of the fact that in this case, and only this, the continued fraction may be written as a sum of partial fractions of the form $\sum_{i=1}^{n} M_{i} /\left(1+x x_{i}\right)$, without a constant term, where $M_{i}>0$, $0<x_{1}<x_{2}<\cdots<x_{n} \leqq 1$. The moments are then $c_{m}=\sum_{i=1}^{n} x^{m} M_{i}$, the function $\phi(u)$ having discontinuities only at the points $x_{i}$.

When the continued fraction does not terminate, it may always be written in the form $1 / a_{1}+x / a_{2}+x / a_{3}+\cdots$, where the $a_{n}$ 's are positive. Then from the work of Stieltjes $[8$, p. 510$]$ we have the theorem that $\phi(u)$ is continuous at $u=0$ if and only if the series $\sum a_{2 n-1}$ diverges. Since

$$
\begin{aligned}
a_{1}= & 1 / c_{0}, a_{3}=g_{1} / c_{0} g_{2}\left(1-g_{1}\right), \cdots \\
a_{2 n+1}= & {\left[g_{1} g_{3} \cdots g_{2 n-1}\left(1-g_{2}\right)\left(1-g_{4}\right) \cdots\left(1-g_{2 n-2}\right)\right] } \\
& \quad \div\left[c_{0} g_{2} g_{4} \cdots g_{2 n}\left(1-g_{1}\right)\left(1-g_{3}\right) \cdots\left(1-g_{2 n-1}\right)\right],
\end{aligned}
$$

this condition appears at once in terms of the parameters $g_{n}$. Remembering that in addition to the continuity of $\phi(u)$ at $u=0$ we require for regularity that $c_{0}=1$, we have the following theorem.

THEOREM 2.1. The totally monotone sequence $\left\{c_{n}\right\}$ is regular if and only if the power series $c_{0}-c_{1} x+c_{2} x^{2}-\cdots$ has a terminating corresponding continued fraction of the form

$$
\frac{1}{1}+\frac{g_{1} x}{1}+\frac{\left(1-g_{1}\right) g_{2} x}{1}+\cdots+\frac{\left(1-g_{2 n-2}\right) g_{2 n-1} x}{1}
$$

where $0<g_{k}<1,(k=1,2,3, \cdots, 2 n-2), 0<g_{2 n-1} \leqq 1$ (in which case the distribution of mass is finite), or else has a nonterminating continued fraction of the form

$$
\frac{1}{1}+\frac{g_{1} x}{1}+\frac{\left(1-g_{1}\right) g_{2} x}{1}+\frac{\left(1-g_{2}\right) g_{3} x}{1}+\cdots,
$$

where $0<g_{n}<1,(n=1,2,3, \cdots)$, and the series

$$
\sum \frac{g_{1} g_{3} \cdots g_{2 n+1}\left(1-g_{2}\right)\left(1-g_{4}\right) \cdots\left(1-g_{2 n}\right)}{g_{2} g_{4} \cdots g_{2 n+2}\left(1-g_{1}\right)\left(1-g_{3}\right) \cdots\left(1-g_{2 n+1}\right)}
$$

diverges (in which case there is an infinite distribution of mass). 
We shall record here for future reference some known properties of the special Stieltjes continued fraction with which we are concerned. These will be stated in the form of theorems, with adequate references.

TheoRem $2.2\left[6\right.$, p. 166]. If $g_{1}, g_{2}, g_{3}, \cdots$ are any real or complex numbers, and $P(x)$ is a power series in ascending powers of $x$ such that

$$
P(x) \sim 1+g_{1} x / 1+\left(1-g_{1}\right) g_{2} x / 1+\left(1-g_{2}\right) g_{3} x / 1+\cdots,
$$

then

$$
(1+x) / P(x) \sim 1+\left(1-g_{1}\right) x / 1+g_{1}\left(1-g_{2}\right) x / 1+g_{2}\left(1-g_{3}\right) x / 1+\cdots .
$$

If $c_{0} \neq 0$ and we put $c_{0} / P(x)=c_{0}-c_{1} x+c_{2} x^{2}-\cdots$, this statement takes the following form: if $c_{0}-c_{1} x+c_{2} x^{2}-\cdots \sim c_{0} / 1+g_{1} x / 1+\left(1-g_{1}\right) g_{2} x / 1+\left(1-g_{2}\right) g_{3} x / 1$ $+\cdots$, then $\Delta c_{0}-\Delta c_{1} x+\Delta c_{2} x^{2}-\cdots \sim \Delta c_{0} / 1+g_{1}\left(1-g_{2}\right) x / 1+g_{2}\left(1-g_{3}\right) x / 1$ $+\cdots$.

TheOREM 2.3 [9, p. 159]. If $g_{1}, g_{2}, g_{3}, \cdots$ are real, $0<g_{1}<1,0 \leqq g_{n}<1$, $n>1$, then the continued fraction

$$
g_{1} / 1+\left(1-g_{1}\right) g_{2} x / 1+\left(1-g_{2}\right) g_{3} x / 1+\cdots
$$

converges uniformly for $|x| \leqq 1$. The function $f(x)$ represented is continuous for $|x| \leqq 1$, analytic for $|x|<1$, and its modulus for $|x| \leqq 1$ does not exceed

$$
1-\left[\sum \frac{g_{1} g_{2} \cdots g_{n}}{\left(1-g_{1}\right)\left(1-g_{2}\right) \cdots\left(1-g_{n}\right)}\right]^{-1} \text {. }
$$

This is the least upper bound, since it is assumed by $f(x)$ at $x=-1$.

The continued fraction converges uniformly over every bounded closed region containing no real point $x$ which is less than or equal to -1 , and $f(x)$ is analytic in every such region [8].

3. The difference matrix. Let $\left\{c_{n}\right\}$ be a totally monotone sequence. Then

$$
c_{n}=\int_{0}^{1} u^{n} d \phi(u)
$$

where $\phi(u)$ is monotone non-decreasing. Now, the $m$ th difference of $c_{n}$ is given by the formula

$$
\Delta^{m} c_{n}=\int_{0}^{1}(1-u)^{m} u^{n} d \phi(u), \quad m, n=0,1,2, \cdots .
$$

If we keep $m$ fixed and allow $n$ to vary, it is evident that the resulting sequence $\left(\Delta^{m} c_{0}, \Delta^{m} c_{1}, \Delta^{m} c_{2}, \cdots\right)$ is totally monotone, the mass function being $\int_{0}^{u}(1-t)^{m} d \phi(t)$. Moreover, if we keep $n$ fixed and allow $m$ to vary we obtain the sequence $\left(c_{n}, \Delta c_{n}, \Delta^{2} c_{n}, \cdots\right)$. Inasmuch as $\Delta^{m} c_{n}=\int_{0}^{1} u^{m}\left[-(1-u)^{n}\right] d \phi(1-u)$, it is clear that this sequence is totally monotone, the mass function being 
$\int_{0}^{u}\left[-(1-t)^{n}\right] d \phi(1-t)$. Thus, the row and column sequences of the difference matrix $\Delta$ are all totally monotone.

Consider next a diagonal sequence

$$
c_{n}, \Delta c_{n+1}, \Delta^{2} c_{n+2}, \cdots,
$$

or

$$
\Delta^{n} c_{0}, \Delta^{n+1} c_{1}, \Delta^{n+2} c_{2}, \cdots
$$

We may write

$$
\Delta^{k} c_{n+k}=\int_{0}^{1 / 4} u^{k} d \phi^{\prime}(u), \quad \Delta^{n+k} c_{k}=\int_{0}^{1 / 4} u^{k} d \phi^{\prime \prime}(u),
$$

where

$$
d \phi^{\prime}(u)=u_{1}^{n} d \phi\left(u_{1}\right)-u_{2}^{n} d \phi\left(u_{2}\right), \quad d \phi^{\prime \prime}(u)=u_{2}^{n} d \phi\left(u_{1}\right)-u_{1}^{n} d \phi\left(u_{2}\right),
$$

and $u_{1}$ is the smallest and $u_{2}$ the largest of the roots of the quadratic equation $u^{2}-u+v=0,0 \leqq v \leqq \frac{1}{4}$. Accordingly we observe that the sequences (3.1) and (3.2) are totally monotone.

An inspection of the mass functions for these row, column, and diagonal sequences reveals that they can all be made regular, by dividing all members of each by its first member, if and only if $\phi(u)$ is continuous at $u=0$ and at $u=1$. In Theorem 2.1 we gave necessary and sufficient conditions for the continuity at $u=0$, in terms of the continued fraction corresponding to the base sequence. We now propose to do likewise for the point $u=1$.

The case of a finite distribution of mass is disposed of by means of the next theorem, which follows from the work of Wall [6].

THEOREM 3.1. Let $\left\{c_{n}\right\}$ be a totally monotone sequence corresponding to a finite distribution of mass, and let $c_{0} / 1+g_{1} x / 1+\left(1-g_{1}\right) g_{2} x / 1+\cdots+\left(1-g_{n-1}\right) g_{n} x / 1$ be the corresponding (necessarily terminating) continued fraction, where $c_{0}>0$, $0<g_{i}<1,(i=1,2,3, \cdots, n-1), 0<g_{n} \leqq 1$. Then the mass function $\phi(u)$ is continuous at $u=1$ if and only if $g_{n}<1$.

Turning to the case of an infinite distribution of mass, we consider the power series $c_{0}-\Delta c_{0} x+\Delta^{2} c_{0} x^{2}-\cdots$ with coefficients from the first column of the difference matrix. Let $c_{0} / 1+h_{1} x / 1+\left(1-h_{1}\right) h_{2} x / 1+\cdots$ be the corresponding continued fraction. Then it is apparent, in view of Theorem 2.1, that $\phi(1-u)$ will be continuous at $u=0$, that is, $\phi(u)$ will be continuous at $u=1$, if and only if the series obtained from (2.3) by replacing $g_{n}$ by $h_{n}$, $(n=1,2,3, \cdots)$, is divergent. The problem will therefore be solved if we determine the $h_{n}$ 's as functions of the $g_{n}$ 's. We shall prove that $h_{2 n}=g_{2 n}$, $h_{2 n-1}=1-g_{2 n-1},(n=1,2,3, \cdots)$. In fact, the following theorem provides a companion theorem to Theorem 2.2. 
THeOREM 3.2. Let $c_{0}$ be different from 0 , and let $g_{1}, g_{2}, g_{3}, \cdots$ be arbitrary real or complex numbers. Then, if

(3.3) $c_{0}-c_{1} x+c_{2} x^{2}-\cdots \sim \frac{c_{0}}{1}+\frac{g_{1} x}{1}+\frac{\left(1-g_{1}\right) g_{2} x}{1}+\frac{\left(1-g_{2}\right) g_{3} x}{1}+\cdots$,

we have

$$
c_{0}-\Delta c_{0} x+\Delta^{2} c_{0} x^{2}-\cdots
$$

$$
\sim \frac{c_{0}}{1}+\frac{\left(1-g_{1}\right) \cdot x}{1}+\frac{g_{1} g_{2} x}{1}+\frac{\left(1-g_{2}\right)\left(1-g_{3}\right) x}{1}+\cdots
$$

where the second continued fraction is obtained from the first by replacing $g_{2 n-1}$ by $1-g_{2 n-1},(n=1,2,3, \cdots)$.

Let

$$
c_{0}-a_{1} x+a_{2} x^{2}-\cdots
$$

$$
\sim \frac{c_{0}}{1}+\frac{\left(1-g_{1}\right) x}{1}+\frac{g_{1} g_{2} x}{1}+\frac{\left(1-g_{2}\right)\left(1-g_{3}\right) x}{1}+\cdots
$$

It is required to prove that $a_{n}=\Delta^{n} c_{0}$. Replace $x$ by $-x$, multiply by $x$, and then replace $x$ by $x /(1+x)$ in the last relation. We obtain

$$
\begin{aligned}
& c_{0}\left(\frac{x}{1+x}\right)+a_{1}\left(\frac{x}{1+x}\right)^{2}+a_{2}\left(\frac{x}{1+x}\right)^{3}+\ldots \\
& \quad \sim \frac{c_{0}}{1+x}-\frac{\left(1-g_{1}\right) x}{1}-\frac{g_{1} g_{2} x}{1+x}-\frac{\left(1-g_{2}\right)\left(1-g_{3}\right) x}{1}-\ldots .
\end{aligned}
$$

Now, the even part of the last continued fraction (that is, the continued fraction having as its sequence of approximants the even approximants of this continued fraction) is the same as the even part of the continued fraction of (3.3). It follows that the formal power series expansion of the left-hand member of (3.6) is $c_{0}-c_{1} x+c_{2} x^{2}-\cdots$ and hence that $a_{n}$ must equal $\Delta^{n} c_{0}$.

With reference to the difference matrix $\Delta$ the principal result of this discussion may conveniently be summarized in the following theorem.

TheOREM 3.3. Let $\left\{c_{n}\right\}$ be a totally monotone sequence corresponding to an infinite distribution of mass. Then the sequence $\left(c_{0}, \Delta c_{0}, \Delta^{2} c_{0}, \cdots\right)$, the first column of $\Delta$, is regular if and only if $c_{0}=1$ and the series

$$
\sum \frac{\left(1-g_{1}\right)\left(1-g_{2}\right) \cdots\left(1-g_{2 n-1}\right)}{g_{1} g_{2} \cdots g_{2 n}}
$$

diverges, where the $g_{n}$ 's are related to the $c_{n}$ 's as in $(1.5$, iii). The sequence 
$\left(c_{0}, \Delta c_{1}, \Delta^{2} c_{2}, \cdots\right)$, the principal diagonal of $\Delta$, is regular if and only if $c_{0}=1$, and both of the series (2.3) and (3.7) are divergent.

We have given conditions for regularity of certain moment sequences, chosen from the difference matrix, in terms of the continued fraction corresponding to the base sequence. The conditions can also be given in terms of the moment generating function

$$
f(x)=\int_{0}^{1} \frac{d \phi(u)}{1+x u} .
$$

In fact, Schoenberg [10] gave necessary and sufficient conditions, in terms of $f(x)$, for the continuity of $\phi(u)$ at an arbitrary point $u=t, 0 \leqq t \leqq 1$. We may therefore state the theorem which follows.

THEOREM 3.4. A totally monotone sequence $\left\{c_{n}\right\}$ with corresponding mass function $\phi(u)$ is regular if and only if $c_{0}=1$ and

$$
\lim _{x \rightarrow \infty} \int_{0}^{1} \frac{d \phi(u)}{1+x u}=0,
$$

where $x \rightarrow \infty$ along any ray except the negative half of the real axis. The sequence $\left(c_{0}, \Delta c_{0}, \Delta^{2} c_{0}, \cdots\right)$ is regular if and only if $c_{0}=0$, and

$$
\lim _{x \rightarrow-1}(1+x) \int_{0}^{1} \frac{d \phi(u)}{1+x u}=0,
$$

where $x \rightarrow-1$ through values interior to or upon the circle $|x|=1$. Finally, the sequence $\left(c_{0}, \Delta c_{1}, \Delta^{2} c_{2}, \cdots\right)$ is regular if and only if $c_{0}=1$, and (3.8), (3.9) both hold.

The regularity conditions can also be given in terms of the moments themselves. Indeed, it is not difficult to show that $\lim _{n \rightarrow \infty} c_{n}=\phi(1)-\phi(1-0)$, and $\lim _{n \rightarrow \infty} \Delta^{n} c_{0}=\phi(+0)-\phi(0)$. Hence we have the following theorem.

Theorem 3.5. The limits (3.8) and (3.9) in Theorem 3.4 may be replaced by the limits

$$
\lim _{n \rightarrow \infty} \Delta^{n} c_{0}=0
$$

and $\lim _{n \rightarrow \infty} c_{n}=0$, respectively.

If the limit (3.10) is $k>0$, then $k$ is the amount of the discontinuity of $\phi(u)$ at $u=0$. If then we subtract $k$ from $c_{0}$ in the sequence $\left\{c_{n}\right\}$, the resulting sequence is totally monotone, and can be made regular by dividing every member by $c_{0}-k$.

Wall [6] considered the class of moment generating functions $f(x)$ $=\sum_{i=0}^{\infty} c_{i}(-x)^{i}$ in which the coefficients $c_{n}$ form a totally monotone sequence. 
In particular, he characterized in several ways the subclass of these functions which are bounded in the unit circle. It is of interest to apply Theorem 3.2 to obtain results of this kind.

THEOREM 3.6. If $f(x)=c_{0} / 1+g_{1} x / 1+\left(1-g_{1}\right) g_{2} x / 1+\left(1-g_{2}\right) g_{3} x / 1+\cdots$, where $c_{0}>0,0<g_{n}<1, n \geqq 1$, so that $f(x)$ is a moment generating function corresponding to an infinite distribution of mass, then the modulus of the function $(1+x) f(x)$ is bounded in the half-plane $\Re(x)>-\frac{1}{2}$ if and only if the series

$$
1+\frac{\left(1-g_{1}\right)}{g_{1}}+\frac{\left(1-g_{1}\right) g_{2}}{g_{1}\left(1-g_{2}\right)}+\frac{\left(1-g_{1}\right) g_{2}\left(1-g_{3}\right)}{g_{1}\left(1-g_{2}\right) g_{3}}+\cdots
$$

is convergent.

If we put $f_{1}(x)=c_{0}-\Delta c_{0} x+\Delta^{2} c_{0} x^{2}-\cdots$, then $(1+x) f(x)=f_{1}(w)$ where $w=-x /(1+x)$. Now $\Re(x)>-\frac{1}{2}$ if and only if $|w|<1$. But $[6$, p. 181] if $f_{1}(w)=c_{0} / 1+h_{1} w / 1+\left(1-h_{1}\right) h_{2} w / 1+\left(1-h_{2}\right) h_{3} w / 1+\cdots$, then the modulus of $f_{1}(w)$ is bounded for $|w|<1$ if and only if the series

$$
\sum\left[h_{1} h_{2} \cdots h_{n} /\left(1-h_{1}\right)\left(1-h_{2}\right) \cdots\left(1-h_{n}\right)\right]
$$

converges. Since by Theorem 3.2, $h_{n}$ equals $g_{n}$ or $1-g_{n}$ according as $n$ is even or odd, it will be seen that the latter series is the same as (3.11).

One may prove that $(1+x) f(x)$ has its modulus bounded for $\Re(x)>-\frac{1}{2}$ if and only if $f(x)$ has a Stieltjes integral representation of the form $\int_{0}^{1} u d \phi(u) /(1+x u)$; and that the moduli of $f(x)$ and of $(1+x) f(x)$ are bounded in the unit circle, and in the half-plane $\Re(x)>-\frac{1}{2}$, respectively, if and only if the integral has the form $\int_{0}^{1} u(1-u) d \phi(u) /(1+x u)$.

4. An illustration. In this section we offer an example to illustrate some of the results of the two preceding sections.

Let $r$ be real, $0<r<1$, and consider the function

$$
f(x)=\frac{1}{1}+\frac{r^{2}(1-r) x}{1}+\frac{r^{3}\left(1-r^{2}\right) x}{1}+\frac{r^{4}\left(1-r^{3}\right) x}{1}+\cdots
$$

We shall determine the moments generated by this function and the corresponding mass function $\phi(u)$.

Put $F(x)=1+r x f(x)$, so that $F(x)=1+r x / 1+r^{2}(1-r) x / 1+r^{3}\left(1-r^{2}\right) x / 1$ $+\cdots$. Then by Theorem 2.2 we have $(1+x) / F(x)=1+(1-r) x / 1$ $+r\left(1-r^{2}\right) x / 1+r^{2}\left(1-r^{3}\right) x / 1+\cdots$ so that $F(x)$ satisfies the functional equation

$$
F(x)=1+\frac{r x}{1+r^{2} x} F\left(r^{2} x\right)
$$

If we put $f(x)=c_{0}-c_{1} x+c_{2} x^{2}-\cdots$, we obtain quite readily the values of the moments $c_{n}$ from this functional relation: 


$$
c_{0}=1, \quad c_{n}=r^{2 n}(1-r)\left(1-r^{3}\right) \cdots\left(1-r^{2 n-1}\right), \quad \cdot n=1,2,3, \cdots .
$$

We obtain the following items of information about the corresponding mass function $\phi(u)$.

(a) Since $\lim _{n \rightarrow \infty} r^{n}\left(1-r^{n-1}\right)=0$, it follows from a result of Stieltjes [8, p. 560] that $f(x)$ is a meromorphic function of $x$. Consequently $\phi(u)$ is a step function (with infinitely many discontinuities).

(b) Since $\lim c_{n}=0, \phi(u)$ is continuous at $u=1$ by Theorem 3.5 .

(c) From a result of Wall $\left[6\right.$, p. 172] there exist numbers $g_{1}, g_{2}, g_{3}, \cdots$, $0<g_{n}<1$, such that $g_{1}=r^{2}(1-r), g_{2}\left(1-g_{1}\right)=r^{3}\left(1-r^{2}\right), g_{3}\left(1-g_{2}\right)=r^{4}\left(1-r^{3}\right)$, ... Then we find that the test-ratio for the series of Theorem 2.1 is

$$
\frac{g_{2 n-1}\left(1-g_{2 n-2}\right)}{g_{2 n}\left(1-g_{2 n-1}\right)}=\frac{1-r^{2 n-1}}{r\left(1-r^{2 n}\right)},
$$

which has the limit $(1 / r)>1$ as $n \rightarrow \infty$. Since the series then diverges, we conclude that $\phi(u)$ is continuous at $u=0$.

(d) In order to locate the discontinuities of $\phi(u)$ we have to locate the poles of $f(x)$. From (4.1) we have the formal expansion

$$
\begin{aligned}
F(x)=1 & +\frac{r x}{1+r^{2} x}+\frac{r^{4} x^{2}}{\left(1+r^{2} x\right)\left(1+r^{4} x\right)}+\cdots \\
& +\frac{r^{n}}{\left(1+r^{2} x\right)\left(1+r^{4} x\right) \cdots\left(1+r^{2 n} x\right)}+\cdots .
\end{aligned}
$$

The series on the right converges for all $x \neq-1 / r^{2 n},(n=1,2,3, \cdots)$, and is uniformly convergent in any bounded region from which the interiors of small circles about these points have been removed. Let $S_{n}$ denote the sum of the first $n$ terms of this series, Then, from (4.1), we have

$$
F(x)=S_{n}+\frac{r^{n^{2}} x^{n}}{\left(1+r^{2} x\right)\left(1+r^{4} x\right) \cdots\left(1+r^{2 n} x\right)} F\left(r^{2 n} x\right), \quad n=1,2,3, \cdots .
$$

Now by Theorem $2.3|F(x)|$ is bounded for $|x| \leqq 1$. Consequently $\lim _{n \rightarrow \infty} S_{n}=F(x)$ for $|x| \leqq 1$. It follows that (4.3) is a valid expansion of the function $F(x)$.

Since $F(x)=1+r x f(x)$, we have

$$
f(x)=\sum_{n=1}^{\infty} \frac{r^{n(n-1)} x^{n-1}}{\left(1+r^{2} x\right)\left(1+r^{4} x\right) \cdots\left(1+r^{2 n} x\right)},
$$

for all $x \neq-1 / r^{2},-1 / r^{4}, \cdots$, and the latter points are the poles of $f(x)$.

(e) From (d) it follows that $f(x)$ must have an expansion of the form

$$
f(x)=\sum_{n=1}^{\infty} \frac{M_{n}}{1+r^{2 n} x}
$$


where $M_{n}>0,(n=1,2,3, \cdots)$. Thus, the function $\phi(u)$ will be completely determined at all points of continuity (except for an additive constant) if we know the values of the numbers $M_{n}$. By (4.1) we have $\left(1+r^{2} x\right) f(x)$ $=1+r^{3} x f\left(r^{2} x\right)$, and therefore

$$
\begin{aligned}
M_{1} & =1-r f(-1)=1-r-r^{3}(1-r)-r^{5}(1-r)\left(1-r^{3}\right)-\cdots \\
& =\prod_{n=1}^{\infty}\left(1-r^{2 n-1}\right) .
\end{aligned}
$$

Then, by this same relation,

$$
M_{n}=\frac{r}{1-r^{2 n-2}} M_{n-1}, \quad n=2,3,4, \cdots .
$$

This determines $\phi(u)$ in accordance with the following table of values.

$$
\begin{aligned}
& \text { Value of } \phi(u) \\
& \text { Value of } u \\
& 1 \\
& r^{2} \leqq u \leqq 1 \\
& 1-M_{1} \\
& r^{4} \leqq u<r^{2} \\
& 1-\left(1+\frac{r}{1-r^{2}}\right) M_{1} \\
& r^{6} \leqq u<r^{4} \\
& 1-\left(1+\frac{r}{1-r^{2}}+\frac{r^{2}}{\left(1-r^{2}\right)\left(1-r^{4}\right)}\right) M_{1} \\
& r^{8} \leqq u<r^{6} \\
& 0 \\
& u=0
\end{aligned}
$$

5. Hypergeometric summability. The continued fraction of Gauss [11, p. 348 ] generates an interesting totally monotone sequence when the parameters are properly restricted. Thus, if we have given the special hypergeometric series

$$
F(\alpha, 1, \gamma, x)=1+\frac{\alpha}{\gamma} x+\frac{\alpha(\alpha+1)}{\gamma(\gamma+1)} x^{2}+\cdots,
$$

we can obtain the representation

$$
F(\alpha, 1, \gamma,-x)=1 / 1+g_{1} x / 1+\left(1-g_{1}\right) g_{2} x / 1+\left(1-g_{2}\right) g_{3} x / 1+\cdots,
$$

where

$$
g_{2 n}=\frac{n}{\gamma+2 n-1}, \quad g_{2 n-1}=\frac{\alpha+n-1}{\gamma+2 n-2}, \quad n=1,2,3, \cdots .
$$

The $g_{n}$ 's will be real and will lie between 0 and 1 if and only if $\alpha$ and $\gamma$ are real, $\gamma>\alpha>0$. The moments are then 


$$
c_{0}=1, \quad c_{n}=\frac{\alpha(\alpha+1)(\alpha+2) \cdots(\alpha+n-1)}{\gamma(\gamma+1)(\gamma+2) \cdots(\gamma+n-1)}, \quad n=1,2,3, \cdots .
$$

Since the hypergometric series converges for $x=1, \gamma>\alpha$, it follows that $\lim c_{n}=0$, and hence that the mass function $\phi(u)$ is continuous at $u=1$. Moreover, it is easy to show that the series (2.3) diverges, and hence that $\phi(u)$ is continuous at $u=0$. Accordingly, the moment sequence generated by this continued fraction is regular when $\alpha, \gamma$ are real and $\gamma>\alpha>0$.

We can determine $\phi(u)$ from the familiar Eulerian integral of the first kind. In fact, we have

where

$$
F(\alpha, 1, \gamma,-x)=\int_{0}^{1} \frac{d \phi(u)}{1+x u}
$$

$$
\phi(u)=\frac{\Gamma(\gamma)}{\Gamma(\alpha) \Gamma(\gamma-\alpha)} \int_{0}^{u} t^{\alpha-1}(1-t)^{\gamma-\alpha-1} d t .
$$

The sequence (5.1) is a special case of the sequence of coefficients in the general hypergeometric series:

$$
\begin{array}{r}
(5.2) c_{n}=\frac{\alpha(\alpha+1)(\alpha+2) \cdots(\alpha+n-1) \beta(\beta+1)(\beta+2) \cdots(\beta+n-1)}{\gamma(\gamma+1)(\gamma+2) \cdots(\gamma+n-1) \cdot 1 \cdot 2 \cdot 3 \cdots n} \\
n=1,2,3, \cdots .
\end{array}
$$

Accordingly, it is convenient to designate the method of summability defined by (5.2), when the sequence is regular, as hypergeometric summability. In this connection we use the symbol $(H, \alpha, \beta, \gamma)$, where in particular the sequence (5.1) defines summability $(H, \alpha, 1, \gamma)$.

By means of (1.4) the general term of the Hausdorff matrix associated with summability $(H, \alpha, 1, \gamma)$ is readily found to be

$$
\frac{C_{\alpha+n-1, n} C_{m-n+\gamma-\alpha-1, m-n}}{C_{\gamma+m-1, m}}, \quad \gamma>\alpha>0 .
$$

Next we display some of the inclusion and equivalence relationships between the hypergeometric methods. Some of the symbolism is not readily intelligible and will be explained presently.

(5.4) (i) $(H, \alpha, 1, \gamma)=(H, 1, \alpha, \gamma), \gamma>\alpha$;

(ii) $(H, \alpha, \gamma, \gamma)=(H, \alpha, 1,1), 0<\alpha<1$; $\gamma>0$;

(iii) $(H, 1,1, \gamma+1)=(C, \gamma), \gamma>0$;

(iv) $(H, \alpha, 1, \gamma+1) \subset(C, \gamma), \alpha>1 ; \gamma>0 ;(H, 1,1, \alpha)$;

(v) $(H, \alpha, 1, \gamma+1) \supset(C, \gamma), 0<\alpha<1$; $\gamma>0$; $(H, \alpha, 1,1)$; 
(vi) $(H, \alpha, 1, \alpha+1) \subset(C, \alpha), \alpha>1$;

(vii) $(H, \alpha, 1, \alpha+1) \supset(C, \alpha), 0<\alpha<1$;

(viii) $\left(H, \alpha, 1, \gamma_{1}\right) \subset\left(H, \alpha, 1, \gamma_{2}\right), \gamma_{2}>\gamma_{1}>\alpha>0 ;\left(H, \gamma_{1}, 1, \gamma_{2}\right)$;

(ix) $\left(H, \alpha_{2}, 1, \gamma\right) \subset\left(H, \alpha_{1}, 1, \gamma\right), \alpha_{2}>\alpha_{1}>0 ; \gamma>0 ;\left(H, \alpha_{1}, 1, \alpha_{2}\right)$;

(x) $\left(C, \alpha_{1}\right) \subset\left(C, \alpha_{2}\right), \alpha_{2}>\alpha_{1}>-1 ;\left(H, \alpha_{1}+1,1, \alpha_{2}+1\right)$;

(xi) $(H, \alpha, 1, \alpha+1) \approx(H, \beta, 1, \beta+1) ; \alpha, \beta>0$;

(xii) $(C, 1) \approx(H, \alpha, 1, \alpha+1), \alpha>0$;

(xiii) $\left(H, \alpha_{1}, 1, \alpha_{1}+\beta\right) \approx\left(H, \alpha_{2}, 1, \alpha_{2}+\beta\right) ; \alpha_{1}, \alpha_{2}, \beta>0 ;\left|\alpha_{1}-\alpha_{2}\right|$ $=1,2,3, \cdots$;

(xiv) $\left(H, \alpha, k_{1}+1, \gamma\right) \supset\left(H, \alpha, k_{2}+1, \gamma\right) ; k_{1}, k_{2}=0,1,2, \cdots ; k_{2}>k_{1}$; $\gamma>\alpha+\kappa_{2}>1 ;\left(H, k_{1}+1,1, k_{2}+1\right)$;

(xv) $(H, \alpha, 1, \gamma) \supset(H, \alpha, k+1, \gamma), k=1,2,3, \cdots ; \gamma>\alpha+\kappa>1$; $(H, 1,1, k+1)$.

It should be mentioned that the matrix defined by (5.3) can be found in the literature on summability, but not in the same form. It was used by Cesàro in his celebrated theorem on the Cauchy product of two Cesàro summable series $[12$, p. 489], by Knopp in his proof of the equivalence of the $(C, k)$ and $(H, k)$ methods for positive integral values of $k[12, \mathrm{p} .481]$, and by Hausdorff [3] in his proof of the same equivalence theorem. However, it has never before been associated with the sequence of coefficients in the hypergeometric series, and most of the relationships in (5.4) are new.

The identities (i) and (ii), and other related ones, obtain in virtue of the symmetric form of the sequence (5.2). The identity (iii) merely exhibits Cesàro summability as a special case of hypergeometric summability.

The remaining relationships are proved with the aid of the following theorems of Hausdorff [4].

THEOREM 5.1. Necessary and sufficient conditions that a matrix

$$
\mathfrak{A}=\mathfrak{D}\left(\delta_{m n} c_{m}\right) \mathfrak{D}=\left(C_{m, n} \Delta^{m-n} c_{n}\right)
$$

be regular are

$$
\begin{aligned}
& c_{0}=1, \\
& \sum_{n=0}^{m} C_{m, n}\left|\Delta^{m-n} c_{n}\right| \leqq M, \quad m=0,1,2, \cdots,
\end{aligned}
$$

$M$ independent of $m$,

$$
\lim _{m \rightarrow \infty} C_{m, n} \Delta^{m-n} C_{n}=0, \quad n=0,1,2, \cdots .
$$

THEOREM 5.2. Let $\mathfrak{A}=\mathfrak{D}\left(\delta_{m n} c_{m}^{A}\right) \mathfrak{D}$ and $\mathfrak{B}=\mathfrak{D}\left(\delta_{m n} c_{m}^{B}\right) \mathfrak{D}$ be regular matrices, and let $\mathfrak{B}^{-1}$ exist. Then, a necessary and sufficient condition that $\mathfrak{A} \supset \mathfrak{B}$ is that the matrix $\mathfrak{D}\left(\delta_{m n} c_{m}^{A} / c_{m}^{B}\right) \mathfrak{D}$ be regular. 
TheOREM 5.3. Let $\mathfrak{A}$ and $\mathfrak{B}$ be regular matrices, and let $\mathfrak{A}^{-1}$ and $\mathfrak{B}^{-1}$ exist. Then, necessary and sufficient conditions that $\mathfrak{A} \approx \mathfrak{B}$ are that the matrices

$$
\mathfrak{D}\left(\delta_{m n} \underset{c_{m}}{\boldsymbol{A}} / c_{m}^{\boldsymbol{B}}\right) \mathfrak{D}, \quad \mathfrak{D}\left(\delta_{m n} \stackrel{\boldsymbol{B}}{c_{m} / \boldsymbol{A}} / \boldsymbol{c}_{m}\right) \mathfrak{D}
$$

be regular.

The relation (iv) is established with the aid of Theorem 5.2. Let $\left\{c_{n}^{\prime}\right\}$ and $\left\{c_{n}^{\prime \prime}\right\}$ be the moment sequences associated respectively with the $(C, \gamma)$ and $(H, \alpha, 1, \gamma+1)$ methods of summation. Then

$$
\begin{aligned}
\frac{c_{n}^{\prime}}{c_{n}^{\prime \prime}} & =\frac{1 \cdot 2 \cdots n}{(\gamma+1)(\gamma+2) \cdots(\gamma+n)} \div \frac{\alpha(\alpha+1) \cdots(\alpha+n-1)}{(\gamma+1)(\gamma+2) \cdots(\gamma+n)} \\
& =\frac{1 \cdot 2 \cdots n}{\alpha(\alpha+1) \cdots(\alpha+n-1)} .
\end{aligned}
$$

However, this defines the regular moment sequence associated with summability $(H, 1,1, \alpha),(\alpha>1)$. This completes the proof, and explains the appendage to statement (iv). The relations (v), (viii), (ix), and (x) are established with the same technique.

The statement $(v)$ is of particular interest due to the scarcity of methods of summation of the type (1.1) which include $(C, \gamma)$ summability. Indeed, we know of only two commonly known methods of the type (1.1) which have this property, the method of de la Vallee Poussin( $\left.{ }^{4}\right)$ and a method of M. Riesz $\left({ }^{5}\right)$. Hille and Tamarkin [15] give an interesting set of necessary and sufficient conditions in order that a Hausdorff method shall include $(C, \gamma)$. They are stated in detail for the case when $\gamma$ is an integer, and the conditions are easily handled.

It is convenient at this time to define Riesz means of the first order and Nörlund means. We write

$$
\begin{gathered}
\frac{p_{0} s_{0}+p_{1} s_{1}+\cdots+p_{n} s_{n}}{P_{n}}, \\
\frac{p_{n} s_{0}+p_{n-1} s_{1}+\cdots+p_{0} s_{n}}{P_{n}},
\end{gathered}
$$

where $P_{n}=p_{0}+p_{1}+\cdots+p_{n},\left(p_{n} \geqq 0\right)$, and $\sum p_{n}$ in (5.5) is always divergent. Since means of the type (5.5) were used in the early development of Riesz typical means, they are called Riesz means and are designated by $\left(R, p_{n}\right)$.

(4) That the method of de la Vallee Poussin includes $(C, \gamma)$ summability was proved independently and virtually simultaneously by T. H. Gronwall $[13$, p. 1664], and C. N. Moore $[14$, p. 1774].

(5) It has been proved that the Riesz logarithmic mean of order $\gamma$ provides a method of summation which includes $(C, \gamma)$ summability [16]. 
Means of the type (5.6) are called Nörlund means and are designated by $\left(N, p_{n}\right)$.

The statements (vi) and (vii) are of course special cases of (iv) and (v) respectively. It is of interest that the transform (1.1) associated with $(H, \alpha, 1, \alpha+1)$, a method of the Riesz type, contains the coefficients in the transform associated with $(C, \alpha)$, a method of the Nörlund type, written in the reverse order.

The statement $(x)$ is a classical result in the domain of Cesàro summability. The proof is particularly easy to understand with the aid of the hypergeometric notation.

In order to prove (xi), (xii), and (xiii) we need the lemma which follows.

LEMMA 5.1. Let the sequences $\left\{c_{n}^{i}\right\},(i=1,2,3, \cdots, k)$, be regular sequences. Then, if $\sum_{i=1}^{k} d_{i}=1$, the sequence $\left\{c_{n}\right\}$, where

is also regular.

$$
c_{n}=\sum_{i=1}^{k} d_{i} c_{n}^{i}, \quad n=0,1,2, \cdots,
$$

The condition imposed on the $c_{i}$ 's insures that condition (i) of Theorem 5.1 be fulfilled. The other conditions of the theorem will be fulfilled since the difference operation is linear.

To start the proof of (xi), let $\left\{c_{n}^{\alpha}\right\}$ and $\left\{c_{n}^{\beta}\right\}$ be the moment sequences associated respectively with the methods $(H, \alpha, 1, \alpha+1)$ and $(H, \beta, 1, \beta+1)$. Then

$$
\frac{c_{n}^{\alpha}}{c_{n}^{\beta}}=\frac{\alpha(\beta+n)}{\beta(\alpha+n)}=\frac{\alpha}{\beta}+\frac{\beta-\alpha}{\beta} \cdot \frac{\alpha}{\alpha+n}, \quad \alpha, \beta>0 .
$$

Let $\left\{c_{n}^{\prime}\right\}=(1,1,1, \cdots)$. Then

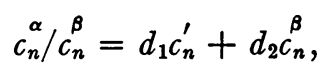

where $d_{1}=\beta / \alpha, d_{2}=(\alpha-\beta) / \alpha, d_{1}+d_{2}=1$. Then, by Lemma 5.1, the sequence $\left\{c_{n}^{\alpha} / c_{n}^{\beta}\right\}$ is regular. Using Theorem 5.2 we now have $(H, \alpha, 1, \alpha+1)$ $\supset(H, \beta, 1, \beta+1)$. By repetition of this argument we can also show that the sequence $\left\{c_{n}^{\beta} / c_{n}^{\alpha}\right\}$ is regular. Thus, we have $(H, \alpha, 1, \alpha+1) \subset(H, \beta, 1, \beta+1)$. This proof is due to Hausdorff [3].

The relation (xii) is an interesting special case of (xi). We observe that summability $(H, \alpha, 1, \alpha+1),(\alpha>0)$, is essentially summability $\left(R, n^{\alpha-1}\right)$, $(\alpha>0)$, and that the relationship $(C, 1) \approx\left(R, n^{\alpha-1}\right),(\alpha>0)$, can be proved in a completely different fashion [17].

Knopp [12, p. 481] has proved (xiii) and (viii) by very laborious methods for integral values of the parameters. These statements afford the entire basis for his Cesàro-Hölder equivalence proof. To establish (xiii) we assume that 
$\alpha_{2}>\alpha_{1}$ and first prove that $\left(H, \alpha_{1}, 1, \alpha_{1}+\beta\right) \approx\left(H, \alpha_{1}+1,1, \alpha_{1}+1+\beta\right)$. Let $\left\{c_{n}^{\prime}\right\}$ and $\left\{c_{n}^{\prime \prime}\right\}$ be the moment sequences associated respectively with these two methods. Then

$$
\begin{aligned}
\frac{c_{n}^{\prime}}{c_{n}^{\prime \prime}} & =\frac{\alpha_{1}}{\alpha_{1}+n} \cdot \frac{\alpha_{1}+\beta+1+n}{\alpha_{1}+\beta+1} \\
& =\frac{\alpha_{1}}{\alpha_{1}+\beta+1}+\frac{\beta+1}{\alpha_{1}+\beta+1} \cdot \frac{\alpha_{1}}{\alpha_{1}+n}, \quad n=0,1,2, \cdots,
\end{aligned}
$$

and is consequently a linear combination of regular sequences, where the constants of combination add up to unity. Likewise, as in the proof of (xi), $\left\{c_{n}^{\prime \prime} / c_{n}^{\prime}\right\}$ is a regular sequence. We use Theorem 5.3 to complete the first stage of the proof. Next we prove in the same fashion that $\left(H, \alpha_{1}+1,1, \alpha_{1}+1+\beta\right)$ $\approx\left(H, \alpha_{1}+2,1, \alpha_{1}+2+\beta\right)$. Clearly, it can readily be established by induction that $\left(H, \alpha_{1}, 1, \alpha_{1}+\beta\right) \approx\left(H, \alpha_{2}, 1, \alpha_{2}+\beta\right)$, provided that $\alpha_{1}$ and $\alpha_{2}$ differ by an integer.

Up to the present time we have considered hypergometric summability $(H, \alpha, \beta, \gamma)$, only for the case $\beta=1, \gamma>\alpha$. It is possible to find non-trivial regular hypergeometric methods at least for positive integral values of $\beta$ by use of a known relationship [18, p. 233] among the hypergeometric functions. If we write

$$
F(\alpha, \beta, \gamma, x)=1+\frac{\alpha \beta}{\gamma \cdot 1} x+\frac{\alpha(\alpha+1) \beta(\beta+1)}{\gamma(\gamma+1) \cdot 1 \cdot 2} x^{2}+\cdots,
$$

the series always converges and represents the function for $|x|<1 ; \alpha, \beta, \gamma>0$. Now, we consider the identity

$$
\begin{aligned}
(\beta-\alpha) F(\alpha, \beta, \gamma, x)+\alpha F(\alpha+1, \beta, \gamma, x)-\beta F(\alpha, \beta+1, \gamma, x) & =0 \\
|x| & <1 .
\end{aligned}
$$

Set $\beta=1$ in (5.7) and write

$$
F(\alpha, 2, \gamma, x)=(1-\alpha) F(\alpha, 1, \gamma, x)+\alpha F(\alpha+1,1, \gamma, x), \quad|x|<1 .
$$

Equating coefficients of $x^{n}$ in this identity we obtain the relation

$$
\frac{C_{\alpha+n-1, n}(n+1)}{C_{\gamma+n-1, n}}=\frac{(1-\alpha) C_{\alpha+n-1, n}}{C_{\gamma+n-1, n}}+\frac{\alpha C_{\alpha+n, n}}{C_{\gamma+n-1, n}}, \quad n=0,1,2, \cdots .
$$

Now, the expression on the right is a linear combination of regular sequences, provided that $\gamma>\alpha+1 ; \alpha>0$, where the constants of combination add up to unity. Consequently, the left member of (5.8) defines a regular method of summability, $(H, \alpha, 2, \gamma), \gamma>\alpha+1 ; \alpha>0$. If we now use (5.7) as a recursion relation, we can define summability $(H, \alpha, 3, \gamma)$ in terms of summability $(H, \alpha, 2, \gamma)$. Continuing this process, we define the regular methods 
$(H, \alpha, k+1, \gamma),(k=0,1,2, \cdots) ; \gamma>\alpha+\kappa ; \alpha>0$, with the associated moment sequence

$$
\frac{C_{\alpha+n-1, n} C_{k+n, n}}{C_{\gamma+n-1, n}}
$$$$
n=0,1,2, \cdots \text {. }
$$

It is now easy to prove the statement (xiv) using methods already established. Statement (xv), a special case of (xiv), clearly indicates the weakness of these newly defined methods.

6. Special methods in the difference matrix. In this section we propose to replace the base sequence $\left\{c_{n}\right\}$ in the difference matrix $\Delta=\left(\Delta^{m} c_{n}\right)$ by known regular sequences whose corresponding mass functions are continuous at $u=1$, and then discuss the resulting methods of summation.

Rows in the difference matrix. If in the matrix $\Delta$ the regular sequence associated with summability $(H, \alpha, 1, \gamma)$ is taken as the base sequence, then the $(k+1)$-st row defines summability $(H, \alpha, 1, \gamma+k)$. It is understood of course, that we normalize each row sequence by dividing each member of the sequence by its first term. Using (5.4, viii) we see that the efficiency of the new methods increases with the depth of the row in the matrix.

If we start with the Euler-Knopp sequence, $\left\{\theta^{n}\right\}, 0<\theta<1$, which defines summability $(E, \theta)$, as the base sequence, no change occurs as a result of repeated differencing.

E. Hille has given us an interesting example to prove that repeated differencing of the base sequence does not always improve or leave unchanged the efficiency of a Hausdorff method corresponding to a monotone non-decreasing mass function $\phi(u)$. In this connection he utilizes the integral

$$
c(z)=\int_{0}^{1} u^{z} d \phi(u), \quad \Re(z) \geqq 0,
$$

which is called the moment function of the associated Hausdorff method. The function $c(z)$ is holomorphic when $\Re(z)>0$, and it is continuous in $\Re(z) \geqq 0$ [15]. To show that a Hausdorff method $\left[H, \phi_{1}(u)\right]$ includes $\left[H, \phi_{2}(u)\right]$ we must establish the existence of a moment function $c(z)$ such that

$$
c_{1}(z)=c(z) c_{2}(z) .
$$

Now, let $\phi(u)$ be a step function with two discontinuities so that

$$
c_{n}=\alpha a^{n}+\beta b^{n}, \quad \alpha+\beta=1, \quad \beta<\alpha ; \quad 0<a<b<1 .
$$

Thus, $\phi(u)$ is a monotone non-decreasing function. The moment function $c(z)=\alpha a^{z}+\beta b^{z}$ has a set of equidistant zeros on a vertical line in the right half-plane. The moment function corresponding to the normalized sequence of the $m$ th row of the difference matrix is $c_{m}(z)=\Delta^{m} c(z) / \Delta^{m} c(0)$. Now, if the Hausdorff method of summation defined by the sequence $\left\{c_{m}(n)\right\}$ is to in- 
clude or be equivalent to the method defined by $\{c(n)\}$, then the quotient $c_{m}(z) / c(z)$ has to be a moment function. In particular, it must be holomorphic for $\Re(z)>0$. However, $c(z)$ vanishes for

$$
z=\left[\log \frac{\alpha}{\beta}+(2 k+1) \pi i\right]\left(\log \frac{b}{a}\right)^{-1}, \quad k=0, \pm 1, \pm 2, \cdots,
$$

and $c_{m}(z)$ vanishes for

$$
\begin{aligned}
z=\left\{\log \left[\frac{\alpha}{\beta}\left(\frac{1-a}{1-b}\right)^{m}\right]+(2 k+1) \pi i\right\}\left(\log \frac{b}{a}\right)^{-1}, \\
k=0, \pm 1, \pm 2, \cdots .
\end{aligned}
$$

Since $(1-a) /(1-b)>1$, the zeros of $c(z)$ and $c_{m}(z)$ are completely distinct, and their quotient is not holomorphic in the right half-plane. This establishes the case in point.

Hille's example raises the problem of determining conditions on the mass function $\phi(u)$ in order that repeated differencing of the base sequence will yield new methods of unchanging or steadily increasing efficiency.

Columns in the difference matrix. If we use the regular moment sequence associated with summability $(C, \alpha)$ as the base sequence in $\Delta$, the $(k+1)$-st column defines summability $(H, \alpha, 1, \alpha+k+1)$. These methods increase in efficiency with increasing $k$. Moreover, from $(5.4$, xiii), we see that if we vary $\alpha$ by any integral amount $\pm p,(p=1,2,3, \cdots)$, such that $\alpha \pm p>0$, the efficiency of the new methods, corresponding to a particular $k$, remains unchanged. If we start with the sequence associated with $(H, \alpha, 1, \gamma)$ as the base sequence, we get summability $(H, \gamma-\alpha, 1, k+\gamma)$ in the $(k+1)$-st column. Again, the efficiency of the methods increases as we traverse the matrix to the right by columns.

Starting with the Euler sequence as a base sequence we get expected results. We obtain the sequence defined by $(1-\theta)^{n}, 0<\theta<1$, in every column. Thus there is no increase in efficiency with an increasing column index.

Diagonal files in the difference matrix. It has already been established that the diagonal files of $\Delta$, as well as the rows and columns, yield regular moment sequences provided the mass function of the base sequence satisfies appropriate continuity requirements. However, starting with familiar base sequences, the diagonal files yield regular moment sequences of a new type. It is of interest to recall that the mass function associated with these new sequences is constant for $\frac{1}{4} \leqq u \leqq 1$. As a result of the discussion in the next section it will be established that all of the diagonal files define methods of summation which include $\left(E, \frac{1}{4}\right)$.

If we start with the sequence associated with summability $(C, \alpha)$ as the base sequence, the principal diagonal then yields the regular moment sequence 


$$
\frac{\alpha}{(n+1) C_{2 n+\alpha, n+1}}, \quad n=0,1,2, \cdots ; \alpha>0 .
$$

If we start with summability $(C, \beta)$ we obtain in the $\nu$ th $u p p e r$ diagonal the regular moment sequence

$$
\frac{\beta C_{\nu+\beta, \beta}}{(n+\nu+1) C_{2 n+\nu+\beta, n+\nu+1}}, \quad n=0,1,2, \cdots ; \beta>0 .
$$

If we start with summability $(C, \gamma)$ the $\mu$ th lower diagonal gives the sequence

$$
\frac{\mu+\gamma}{(n+1) C_{2 n+\mu+\gamma, n+1}}, \quad n=0,1,2, \cdots ; \gamma>0 .
$$

Observe that $\mu$ and $\nu$ vary through positive integral values only. If $\alpha, \beta, \gamma$ also vary through only positive integral values, all of the methods of summation defined by the sequences (6.1), (6.2), and (6.3) are equivalent. Indeed, these methods still remain equivalent to each other if $\alpha, \beta, \gamma$ vary in any fashion so as to differ from each other only by integral amounts. These statements may be proved by using the same technique as employed in proving (5.4, xiii). To illustrate the procedure used, let $\left\{c_{n}^{\prime}\right\}$ and $\left\{c_{n}^{\prime \prime}\right\}$ be respectively the sequence (6.3) for fixed $\mu$ and $\gamma$, and the sequence obtained from (6.3) by replacing $\gamma$ by $\gamma+1$. We propose to show that the methods of summation defined by these sequences are equivalent. We form the quotient

$$
\frac{c_{n}^{\prime}}{{c_{n}^{\prime \prime}}^{\prime \prime}}=\frac{\mu+\gamma}{n+\mu+\gamma} \cdot \frac{n+\frac{1}{2}(\mu+\gamma+1)}{\frac{1}{2}(\mu+\gamma+1)} .
$$

In the light of past experience, we see that both $\left\{c_{n}^{\prime} / c_{n}^{\prime \prime}\right\}$ and $\left\{c_{n}^{\prime \prime} / c_{n}^{\prime}\right\}$ are regular sequences. Thus, the methods of summation in question are equivalent.

Starting with the Knopp-Euler sequence as a base sequence, we obtain the regular sequence $\left\{(1-\theta)^{n} \theta^{n}\right\}, 0<\theta<1$, in all the diagonals. This is clearly another Knopp-Euler sequence.

Symmetry in the difference matrix. It is of some interest to find regular sequences which give rise to symmetry about the principal diagonal of the difference matrix. For the hypergeometric method $(H, \alpha, 1, \gamma)$ we have

$$
\Delta{ }^{m} c_{n}=\frac{C_{\alpha+n-1, n} C_{\gamma-\alpha+m-1, m}}{C_{\gamma+m+n-1, m+n}}
$$

Thus, the method $(H, 1,1, \gamma)=(C, \gamma-1),(\gamma>1)$, and the method $\left(H, \frac{1}{2}, 1,1\right)$ have this property. Moreover, the Knopp-Euler method $\left(E, \frac{1}{2}\right)$ also has this property. 
We first noticed the symmetry of the method $\left(H, \frac{1}{2}, 1,1\right)$ while considering the periodic continued fraction

$$
\frac{1}{1}+\frac{r x}{1}+\frac{r(1-r) x}{1}+\frac{r(1-r) x}{1}+\cdots, \quad 0>r>1 .
$$

The function represented by this continued fraction is

$$
f(x)=\frac{(1-2 r)-[1+4 r(1-r) x]^{1 / 2}}{-2 r(1+x)}=c_{0}-c_{1} x+c_{2} x^{2}-\cdots .
$$

Then

$$
\Delta c_{0}-\Delta c_{1} x+\Delta c_{2} x^{2}-\cdots=\frac{1-[1+4 r(1-r) x]^{1 / 2}}{-2 r x}
$$

Hence

$$
\Delta c_{n}=\frac{(2 n) !}{n !(n+1) !} r^{n}(1-r)^{n+1}, \quad n=0,1,2, \cdots .
$$

The moment sequence (6.4) for $r=\frac{1}{2}$, when normalized, is the moment sequence for summability $\left(H, \frac{1}{2}, 1,1\right)$.

It is easy to prove by means of Theorem 3.2 that the difference matrix is symmetric about the principal diagonal if and only if the function $f(x)$ which generates the base sequence has a continued fraction of the form given in $\left(1.5\right.$, iii) in which $g_{2 n-1}=\frac{1}{2},(n=1,2,3, \cdots)$.

7. Analytic continuation. This section is devoted to some remarks concerning the effectiveness of the Hausdorff methods in the analytic continuation of a power series $\sum a_{n} z^{n}$ outside of its conventional circle of convergence. The Hausdorff methods of particular interest in this respect are those for which the mass function $\phi(u)$ is a monotone non-decreasing function which is constant in the neighborhood of $u=1$.

The Hausdorff transform of the sequence $\left\{s_{n}\right\}$ is given by

$$
\sigma_{m}=\sum_{n=0}^{m} C_{m, n} \Delta^{m-n} C_{n} \cdot s_{n}=\int_{0}^{1} \sum_{n=0}^{m} C_{m, n} u^{n}(1-u)^{m-n} s_{n} d \phi(u) .
$$

If $\phi(u)$ is a monotone non-decreasing function satisfying the regularity conditions (5.5), which is constant for $\delta<u \leqq 1$, we shall designate (7.1) the $\delta$-transform of the sequence $\left\{s_{n}\right\}$.

Now, from a result of Hille and Tamarkin [15], a necessary and sufficient condition that $[H, \phi(u)] \supset(E, \delta)$ is that $\phi(u)=1, \delta \leqq u \leqq 1$. Thus, the $\delta$-method has at least the same efficiency as the Euler-Knopp method in the problem of analytic continuation. It will be of interest to recall the nature of this region of convergence. Corresponding to each singularity $\zeta$ of the power 
series, draw the circle whose equation is

$$
\left|\frac{z}{\zeta}+\frac{1-\delta}{\delta}\right|=\frac{1}{\delta} .
$$

These circles are tangent to the sides of the Borel polygon of summability at the points of singularity. The figure thus constructed is called the curvilinear polygon $B_{\delta}$.

Knopp [19] has established $B_{\delta}$ as the region of convergence for the method $(E, \delta)$ for the case that $\delta=2^{-p},(p=1,2,3, \cdots)$. Using the methods of Knopp, we can readily prove that the $\delta$-transform of $f(z)=\sum a_{n} z^{n}$ converges to $f(z)$ at a point $z$ inside of $B_{\delta}$, but we have been unable to establish divergence outside $B_{\delta}$. However, we shall offer some evidence in support of the following conjecture: a necessary and sufficient condition that a Hausdorff method of summation, corresponding to a monotone non-decreasing $\phi(u)$ satisfying the regularity conditions (1.3), shall sum a power series outside of its circle of convergence is that $\phi(u)$ be constant in the neighborhood of $u=1$.

In support of our conjecture we shall first prove that if $\phi(u)$ is not constant in the neighborhood of $u=1$ then the $\delta$-transform of the geometric series $\sum z^{n}$ diverges for $z=-1-\epsilon, \epsilon>0$. We have for this case

$$
\begin{aligned}
\sigma_{m} & =\frac{1}{1-z}-\frac{z}{1-z} \int_{0}^{1} \sum_{n=0}^{m} C_{m, n}(u z)^{n}(1-u)^{m-n} d \phi(u) \\
& =\frac{1}{1-z}-\frac{z}{1-z} \int_{0}^{1}(u z+1-u)^{m} d \phi(u) .
\end{aligned}
$$

If $z=-1-\epsilon$, then

and

$$
\sigma_{m}=\frac{1}{2+\epsilon}+\frac{1+\epsilon}{2+\epsilon} \int_{0}^{1}(1-2 u-\epsilon u)^{m} d \phi(u),
$$

$$
\left|\sigma_{m}-\frac{1}{2+\epsilon}\right|=\frac{1+\epsilon}{2+\epsilon}\left|\int_{0}^{1}(1-2 u-\epsilon u)^{m} d \phi(u)\right| .
$$

Let $m$ be even. Then, for $\epsilon_{1}>0, \epsilon_{1}<\epsilon, \eta=\left(2+\epsilon_{1}\right) /(2+\epsilon)$, we have

$$
\left|\sigma_{m}-\frac{1}{2+\epsilon}\right|>\frac{1}{2} \int_{\eta}^{1}\left(1+\epsilon_{1}\right)^{m} d \phi(u)=\left(1+\epsilon_{1}\right)^{m}[\phi(1)-\phi(\eta)] .
$$

Hence, $\left|\sigma_{m}\right| \rightarrow \infty$ as $m \rightarrow \infty$.

Next, we shall sum the series $\sum z^{n}$ with a special $\delta$-method. Incidentally, the regular sequences (6.1), (6.2), and (6.3) define $\delta$-methods. Other examples are readily constructed. As a case in point, let $\phi(u)=u / \delta, 0 \leqq u \leqq \delta$, 
$0<\delta<1 ; \phi(u)=1, \delta \leqq u \leqq 1$. Let us test the corresponding $\delta$-transform on the series $\sum z^{n}$. We have

$$
c_{n}=\frac{1}{\delta} \int_{0}^{\delta} u^{n} d u=\frac{\delta^{n}}{n+1}
$$

and

$$
\Delta^{m-n} c_{n}=\frac{1}{\delta} \int_{0}^{\delta} u^{n}(1-u)^{m-n} d u
$$

Then, the $\delta$-transform of the series $\sum z^{n}$ is

$$
\begin{aligned}
\sum_{n=0}^{m} C_{m, n} \Delta^{m-n} C_{n} s_{n} & =\frac{1}{1-z}-\frac{z}{\delta(1-z)} \int_{0}^{\delta} \sum_{n=0}^{m} C_{m, n}(u z)^{n}(1-u)^{m-n} d u \\
& =\frac{1}{1-z}+\frac{z(\delta z+1-\delta)^{m+1}}{\delta(1-z)^{2}(m+1)}-\frac{z}{\delta(1-z)^{2}(m+1)}
\end{aligned}
$$

Clearly the transform converges to $1 /(1-z)$ as $m \rightarrow \infty$ whenever

$$
\left|z+\frac{1-\delta}{\delta}\right|<\frac{1}{\delta}
$$

and diverges to $+\infty$ whenever $|z+(1-\delta) / \delta|>1 / \delta$. Evidently, the region $B_{\delta}$ is the largest region of convergence for the special case under consideration. Finally, the fact that the inequality (7.2) becomes $|z|<1$ as $\delta \rightarrow 1$ is consistent with our conjecture.

\section{REFERENCES}

1. L. L. Silverman, On the Definition of the Sum of a Divergent Series, University of Missouri Studies, Mathematics Series, vol. 1, no. 1, 1913.

2. O. Toeplitz, Über allgemeine lineare Mittelbildungen, Prace Matematyczno-fizyczne, vol. 22 (1911), pp. 131-119.

3. F. Hausdorff, Summationsmethoden und Momentfolgen, I and II, Mathematische Zeitschrift, vol. 9 (1921), pp. 74-1099, 280-299.

4. H. L. Garabedian, Hausdorff matrices, American Mathematical Monthly, vol. 46 (1939), pp. $390-410$.

5. F. Hausdorff, Über das Momentenproblem für ein endliches Interval, Mathematische Zeitschrift, vol. 16 (1923), pp. 220-248.

6. H. S. Wall, Continued fractions and totally monotone sequences, these Transactions, vol. 48 (1940), pp. 165-184.

7. T. J. Stieltjes, Recherches sur les fractions continues, Annales de l'Université de Toulouse, vol. 8, J (1894), pp. 1-122; vol. 9, A (1895), pp. 1-47.

8. T. J. Stieltjes, Oeuvres, vol. 2, Société Mathématique d'Amsterdam, Groningen, P. Nordhoff, 1918.

9. W. T. Scott and H. S. Wall, A convergence theorem for continued fractions, these Transactions, vol. 47 (1940), pp. 155-172.

10. I. J. Schoenberg, Über die asymptotische Verteilung reeller Zahlen mod 1, Mathematische Zeitschrift, vol. 28 (1928), pp. 171-199.

11. O. Perron, Die Lehre von den Kettenbrïchen, Leipzig and Berlin, Teubner, 1913. 
12. K. Knopp, Theorie und Anwendung der unendlichen Reihen, Berlin, Springer, 1922, 2d edition, 1924.

13. T. H. Gronwall, Comptes Rendus de l'Académie des Sciences, Paris, vol. 158 (1914), p. 1664.

14. C. N. Moore, ibid., vol. 158 (1914), p. 1774.

15. E. Hille and J. D. Tamarkin, Questions of relative inclusion in the domain of Hausdorff means, Proceedings of the National Academy of Sciences, vol. 19 (1933), pp. 573-577.

16. G. H. Hardy and M. Riesz, The General Theory of Dirichlet's Series, Cambridge Mathematical Tracts, no. 18, 1915.

17. H. L. Garabedian and W. C. Randels, Theorems on Riesz means, Duke Mathematical Journal, vol. 4 (1938), pp. 529-533.

18. J. Pierpont, The Theory of Functions of a Complex Variable, vol. 2, New York, Ginn, 1912.

19. K. Knopp, Über das Eulersche Summierungsverfahren I, Mathematische Zeitschrift, vol. 15 (1922), pp. 226-253; II, ibid., vol. 18 (1923), pp. 125-156.

NORTHWESTERN UNIVERSITY,

Evanston, Ill. 